former disease, (valvular scar) or trauma, the individual may suffer from acute rheumatic fever, chronic arthritis, myositis, chronic infectious endocarditis, pneumonia, ulcer of stomach, cholecystitis or pancreatitis, respectively dependent on the phase of mutation in pathogenicity, and affinity of the strain of the streptococcus-pneumococcus group in the focus of infection. These principles are important because proper management may $(a)$ prevent the systemic disease and $(b)$ guide one in the proper treatment of the systemic disease. The recognition and removal of chronic foci anywhere in the body is imperative as a prevention of progressive ill health. Emphasis should be made of chronic alveolar abscess, often unrecognized by the patient. Film roentgenograms of the jaws is often the only means of recognition. A pathologic tonsil is a menace and should be wholly removed. Chronic cholecystitis and chronic appendicitis should be promptly treated surgically. Chronic sinusitis may exist for years and finally be an important factor in the development of systemic disease. Following the removal of the focal infection, adequate individual and general hygiene will improve the body defenses and health will be maintained.

The removal of the focus of infection is demanded as a fundamental principle, in the treatment of the systemic diseases especially of the chronic type. Thereafter the management must be individual, based on the character and location of the infection. The attempt to immunize the patient against the infection must be attempted by restorative measures-food, pure air, passive and active graduated exercise, hematinic and other tonics, optimistic surroundings, etc. Autoge:ous vaccines may be used with rational reservation.

122 South Michigan Boulevard.

\section{THE NEWER BACTERIOLOGY OF VARIOUS INFECTIONS AS DETERMINED BY SPECIAL METHODS *}

\section{E. C. ROSENOW, M.D. CHICAGO}

The bacteriology of most infections as worked out heretofore is largely the bacteriology of the fluids of the body and semifluid exudates. In acute infections a bacteriologic study of the fluids commonly yields trustworthy results, but in many chronic conditions the cultures are usually sterile and demonstration of bacteria in the tissues usually fails.

In this short paper I wish to describe methods for making cultures particularly from excised tissues and from the blood and other fluids in which due regard is paid to the question of oxygen pressure, particularly in the primary culture, and to detail very briefly the results obtained by their use in various infections.

\section{PREPARATION OF THE EMULSION FROM THE TISSUES}

The tissues are excised with strict aseptic precautions usually under local anesthesia and cultures made at once, a portion being reserved for microscopic study. In addition to the great care used to avoid contamination from skin or air the surface of the part used for cultures is sterilized by dipping it into boiling water, or even better, into a vigorous Bunsen flame,

* Read before the Section on Practice of Medicine at the Sixty-Fifth Annual Session of the American Medical Association, Atiantic City N. J., Junc, 1914 or seared with a hot blade, then rapidly cooled in sterile salt solution and emulsified in the specially devised sterile air-chamber or "tissue-crusher" shown in the accompanying illustrations. The amount of heat used is determined by the size of the tissue at hand. Whenever possible, pieces of tissue at least 2 to $3 \mathrm{~cm}$. in diameter are used for cultures.

The chamber (Fig. 1) is somewhat similar to the one already described ${ }^{1}$ except that it contains a larger mortar and a small-sized meat-chopper. ${ }^{2}$ It consists of an air-tight tin can (19 by $23 \mathrm{~cm}$.) containing a large $(10 \mathrm{~cm}$.) hole on one end with a flanged rim to which is tied a long-sleeved, heavy, fleece-lined canvas glove; there is a smaller side opening $(3 \mathrm{~cm}$.) which is plugged with cotton and a large opening ( 8 by $12 \mathrm{~cm}$.) at the top which is covered with mica and held in place by circular wires and surgeons' adhesive plaster. The meat-chopper is held in place through a hole at the farther end through which the shaft extends and a hole at the bottom through which a thumb-screw fits whereby it is clamped to a wood extension, fastened to a semicircular wood board nailed to the end. On the inside is fastened a suitable-sized tin can which serves as the mortar holder. The container without the meat-chopper answers very

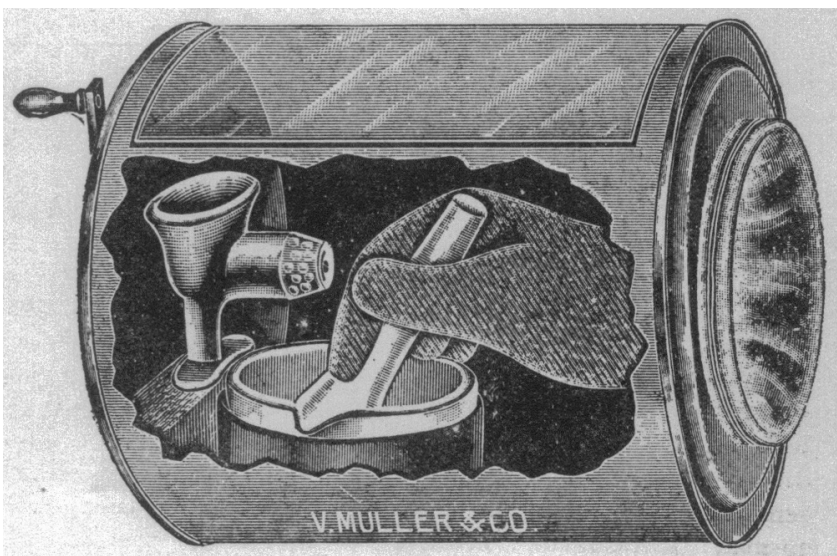

Fig. 1.-Sterile ain chamber. The right hand is used inside the apparatus.

well for small or relatively soft tissues, such as the lymph-glands in arthritis, while the one containing the meat-chopper is preferable for emulsifying larger and firmer tissues such as the thyroid gland, cystic ovaries and the glands in Hodgkin's disease. Sterilization is done in dry heat at $160 \mathrm{C}$. (320 F.) for one hour. For actual use the right hand and forearm are thoroughly scrubbed with soap and water and soaked in $1: 1,000$ mercuric chlorid and then introduced into the container and glove. Alcohol should not be used in steri1izing the hand bec.use the vapors are apt to ignite with explosive violence when the side opening is flamed after removing the cotton plug; the mouth of the tube or bottle containing the tissue is flamed and held near the small opening in the side and the tissue grasped with long sterile forceps, and with the least possible exposure to the room air, inserted into the container and either cut into small pieces with the scissors or passed through the meat-chopper. Great care is exercised at all times not to move the gloved hand in or out of the container while the cotton plug is removed.

1. Rosenow: Centralbl. f. Bakteriol., Orig., 1914, Ixxiv, 366. 2. The meat-chopper is an inexpensive commercial article designated the "Baby Masticator" and manufactured by the Enterprise Manufacturing Company, 176 North Dearborn Street, Chicago. 
After grinding the tissue in the mortar for a time, sterile quartz-sand and the emulsifying fluid, usually dextrose broth, are poured into the mortar through the side opening from thoroughly flamed test-tubes. The cotton plug is reinserted and the emulsifying process is continued. The emulsion when completed is poured into a thoroughly flamed test-tube or graduate of suitable size, inserted through the side opening.

In attempting to simplify as much as possible the emulsification of tissues and to check up the results obtained with the sterile air-chamber, the "tissuecrusher" (Fig. 2), a modification of the method used by Hirschfelder to obtain tissue juice, was devised. It consists of two close-fitting threaded metal tubes; the outer tube is fitted with an air-tight removable bottom, the inner surface being made rough, and a four-sided rim, while the inner tube is fitted with a removable disk containing numerous very small drilled holes and a handle which serves as a wrench. The inner tube is plugged with cotton and placed inside

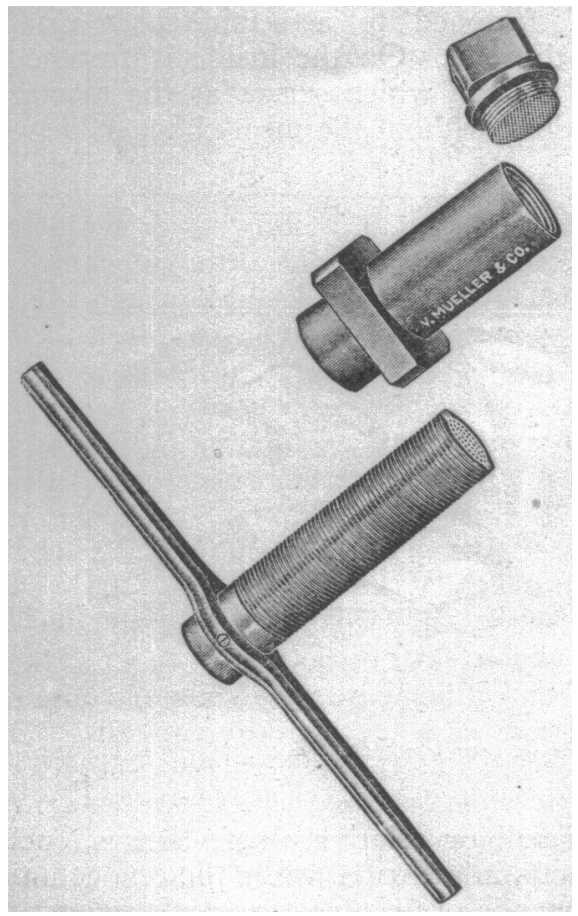

Fig. 2.-Tissue crusher.

the other and the instrument sterilized in hot air. In order to prevent leakage the thread of the inner tube should be covered with hot sterile paraffin or petroleum oil just before using. The tissue is placed in the outer tube and the inner tube turned in the outer, using the necessary force until the tissue is crushed and expressed into the inner tube. The instrument is now held slantwise, the cotton plug flamed and removed, the emulsifying fluid added and the emulsion transferred to a sterile test-tube with a sterile pipet. ${ }^{3}$

\section{METHOD OF PREPARING THE BLOOD-CULTURES}

In order to furnish bacteria from the blood and other sources a gradient of oxygen pressure, the following method for making blood-cultures has been used with good results : From 15 to 30 c.c. of blood are

3. The sterile air-chamber may be made in the laboratory, while the tissue-crusher requires a well equipped machine-ehep for its construcAvenue, Chicago. withdrawn from the vein at the bend of the elbow in the usual way and decalcified, by adding it to a solution of 2 per cent. sodium citrate in sodium chlorid solution in the proportion of 5 c.c. of blood per 1 c.c. of solution. Control tests have shown that the bacteria contained in the blood from a wide range of conditions live in this solution for at least seventy-two hours. The citrate solution is kept in a tube of suitable size closed by an aluminum screw-top containing a rubber disk and sealed with paraffin. This may be used at the bedside and then sent to the laboratory for cultures in case, for some reason, it is not convenient to make the complete culture at the bedside. In my work a portion of the citrated blood has been at once planted and the remainder added by means of a sterile pipet to sterile distilled water contained in bottles which serve as centrifuge tubes, there being at least 10 parts of water to 1 part of citrated blood. This is centrifuged at once and the supernatant hemoglobin containing water is poured off or the sediment removed with a sterile pipet, and planted. The sterile water serves as a means of getting rid of the hemoglobin and the serum. Control tests with the serum after the corpuscles have separated by gravity show that in the aerobic cultures the inhibiting action of the hemoglobin is more marked than that of the serum; in fact, the serum is a desirable constituent in streptococcal cultures. Hence in suspected streptococcal infections the serum and the sediment of the dissolved corpuscles may be planted separately. In some instances the hemoglobin has been converted into methemoglobin by passing carbon monoxid (illuminating gas) through the decalcified blood by means of a sterile pipet containing a long sterile cotton plug.

\section{THE PREPARATION OF THE CULTURES}

The emulsion of the tissues or the sediment of the dehemoglobinated blood is inoculated in the way best suited for the particular case at hand. Shake-cultures in tall columns (10 to $20 \mathrm{~cm}$.) of ascites dextrose ( 1 per cent.) agar and plain agar ( 0.5 to 0.8 per cent. acid to phenolphthalein) with or without serum have yielded the best results. The ascites fluid is collected in a sterile manner and heated on successive days to 60 C. $(140 \mathrm{~F}$.) for one hour or for twenty-four hours and used only after the sterility has been determined. To 9 parts of 0.5 per cent. agar, prepared from Liebig's extract of beef and Witte peptone, 1 part of ascites fluid is added with a sterile pipet after the agar is either thoroughly boiled in a bath or steamed in an Arnold sterilizer for at least fifteen minutes to drive off molecular oxygen, and cooled to $60 \mathrm{C}$. The emulsion or other material is added to the agar when cooled to $39 \mathrm{C}$. (102.2 F.) by inserting the mouth of the pipet to the bottom and forcing out its contents while the pipet is withdrawn. After the cotton plug is flamed and replaced in the test-tube the further mixing is accompanied by partially inverting the tube once or twice. Care is exercised not to blow bubbles of air through the agar.

Owing to the oxygen-consuming power of living cells the bottom of the tubes is rendered anaerobic while the top necessarily remains aerobic, and the space between represents a gradient of oxygen pressure between the two extremes. In order to bring about a still greater range of conditions in view of the consideration that the emulsion or tissue juice may be bactericidal or may favor the growth of the bacteria, 
crily a small amount of enulsion is added to some tubes and a large amount to others. In the case of the blood-sediment or joint fluid when only a small amount is at hand, pieces of sterile rabbit or guinea-pig tissue are added to the bottom of the tube if strict anaerobic conditions are desired. A gradient of $\mathrm{H}$ and $\mathrm{OH}$ ions in the mediums, obtained by superimposing a plus 1 agar or a minus 1 agar and vice versa has also been found useful in some conditions.

Plain serum or ascites, agar and broth, ascitesdextrose-broth in tall tubes, enriched ascites fluid, with or without sterile tissue the surface of Loeffler's serum, plain and dextrose blood-agar slants and plates, kept under aerobic and anaerobic conditions, and at times litmus-milk and ox-bile, and in Hodgkin's disease Dorset's egg mediums are also inoculated. These, while not as favorable culture mediums, have yielded positive results in isolated instances when the shakecultures in agar were negative. The cultures are incubated at $37 \mathrm{C}$. for ten to fourteen days before they are discarded. The tubes of dextrose-agar are carefully examined for colonies in a strong light. Smears are made from the water of condensation of the Loeffler's serum and blood-agar slants, of the liquid cultures if turbid and of the sediment always, and from the bottom of the tube of agar even though no colonies are found. Colonies in the depths of the dextrose-agar are examined by breaking the tube crosswise by means of a glass cutter and a red-hot bead at the end of a glass pipet, the surface of the tube being sterilized in the flame just before breaking it. If a positive result is obtained, at least one of the subcultures is made in such a way as to reproduce as nearly as possible the conditions under which the original growth was obtained. This point is particularly important with those strains which show a preference for anaerobic conditions. Search for unknown bacteria was made in smears stained in various ways from the cut surface of the glands and various emulsions and from the depths of the cultures both by staining and with the aid of dark-field illumination.

\section{RESULTS}

By this method, which is similar to those employed by Theobald Smith and Noguchi but in which there is afforded not only aerobic and anaerobic conditions but a gradation of oxygen pressure between these two points, a gradation of $\mathrm{H}$ and $\mathrm{OH}$ ions, and a wide range of nutrition, positive cultures have been obtained frequently while cultures made in the usual way remained sterile.

Thus certain non-hemolyzing streptococci have been isolated from the blood repeatedly when the usual method failed or showed much fewer colonies; in leukemia this has happened as long as four months before death; in rheumatic fever during the height of the disease in 5 of 9 attempts; in peliosis rheumatica in 2 cases, and occasionally from arthritis deformans. Hemolytic streptococci were obtained from three cases of puerperal sepsis; the Streptococcus virialans in two cases of chronic infectious endocarditis, while the results of the usual cultures suggested the "bacteriafree stage" (Libman) of the disease. Diphtheroid-like organisms have been isolated from the blood during the febrile period in four cases of Hodgkin's disease, and the same organism from the blood as from the nodes in two of three cases of erythema nodosum in which blood-cultures were made. Diphtheroid bacilli have been isolated from the blood in four cases of severe infections resembling rheumatism; the Bacillus aerogenes capsulatus ( $B$. welchii) from the blood in one case of bronchopneumonia, one case of acute thet1matism, and in leukenia.

Streptococci corresponding to those which I have isolated previously from the joints have been isolated from glands draining the involved joints in twelve cases and $B$. zelchii alone or in conjunction with streptococci in four of twelve cases of acute articular rheumatism.

In 54 cases of arthritis deformans one or more glands draining the joints have been examined in each case. Non-hemolyzing streptococci producing usually no distinct green on blood-agar plates and often showing a marked preference for anaerobic conditions in the primary culture but otherwise resembling Streptococcus viridans have been isolated 32 times; staphylococcus 5 times; an organism resembling $B$. mucosus 3 times, Micrococcus catarrhalis and the gonococcus once each, $B$. welchii in 14 cases, and diphtheroid bacilli in 5 cases. In 7 cases the cultures remained sterile. In both rheumatic and deforming arthritis the same organisms have been isolated simultaneously from the fluid or capsule of the joints and the excised gland.

A diphtheroid bacillus showing a wide range of preference for oxygen in the different cases has been cultivated in all of some 40-odd cases of Hodgkin's disease; a staphylococcus in 17 ; Streptococcus viridans in $7 ; B$. welchii in 14 , and an unidentified Gramnegative motile bacillus in 6 cases.

A non-hemolyzing, sometimes green-producing (blood-agar) streptococcus has been obtained from the depth of the tissue in 16 of 21 chronic indurated duodenal and gastric ulcers, and from the small glands adjacent to the ulcer in 4 of 5 cases. A staphylococcuslike organism was present usually in smaller numbers in 16 ; Staphylococcus aureus only once. The base of the ulcers was either entirely sterile or contained only a small number of bacteria and yeast-cells. The number of colonies of streptococci was roughly proportionate to the gross or microscopic evidence of inflammation. Only one perforating ulcer was examined and this showed the largest number of colonies of streptococci. The negative results obtained were in ulcers which seemed to be practically healed. $B$. welchii was isolated from the ulcers in 6 cases and from the adjacent glands in 3 . Three ulcers showed diphtheroid bacilli, 4 yeast-cells, and in a number there were found a few colonies of a large Gram-positive aerobic bacillus, probably belonging to the subtilis group. The colon bacillus was found only once in the simple ulcers excised at operation, but was present in large numbers in one examined shortly after death and in small numbers in two out of three ulcers the seat of carcinoma.

Streptococci quite similar to those from the ulcers have been isolated in pure culture from the excised tissue of the gall-bladder in cholecystitis in one case and from the center of gall-stones in 4 of 7 cases. $B$. welchii and $B$. coli were found together with the streptococcus in the gall-stones in one case and a bacillus resembling closely the typhoid bacillus in another.

A polymorphous, sometimes clubbed, diplobacillus was obtained in pure culture from excised nodes in each of 7 cases of erythema nodosum; from a cervical lymph-gland draining an infected tonsil in one case 
and in mixture from the focus of infection (tonsil and superficial ulcer on anterior pillar) in 2 cases of erythema nodosum.

An anaerobic Gram-positive diplobacillus-like organism has been isolated from the thyroid gland removed at operation in 25 out of 32 cases of goiter (mostly exophthalmic goiter) in man, from the blood on two occasions in a severe case of exophthalmic goiter, and in conjunction with Dr. Moon, in 8 of $12 \mathrm{dogs}$ having goiter. We failed to obtain the organism from the gland in six of seven dogs showing apparently normalsized thyroid glands, while one showed a few colonies. $B$. welchii was found in all but 6 of the thyroid glands examined. Three of these were normal glands in dogs. A staphylococcus which usually produces hemolysis on blood-agar and grows unusually well anaerobically was found in most of the goiters in those from both man and dog. It is as yet impossible to state whether or not this coccus comes from the skin, although it perhaps does not. Non-hemolyzing streptococci have been isolated in 3 goiters from man and in 2 goiters from dogs.

Cystic ovaries from 25 patients have been examined by this method. Streptococci, often good green-producing, have been found in pure culture in 14, in some instances, both from the cyst fluid and the emulsified tissues; but the cyst content is usually sterile. A partially anaerobic diplobacillus resembling quite closely the organism from the thyroid gland has been found in 4; staphylococci in small numbers in 3 ; $B$. avelchii in all but 5 cases.

\section{SIGNIFICANCE OF THE FINDINGS}

The common localization and lesions produced on intravenous injection of streptococci soon after isolation from acute rheumatism, from arthritis deformans, from ulcer of the stomach and from the tissue of the gall-bladder in cholecystitis and the center of gallstones, together with other facts, suggests strongly that they are not secondary invaders but the important etiologic factor in each of these conditions because the strains from rheumatism commonly produce arthritis, endocarditis, myocarditis and pericarditis; the strains from arthritis deformans, arthritis and myositis but rarely endocarditis or pericarditis; the strains from the depths of ulcers of the stomach often show a striking affinity for the mucous membrane of the stomach and duodenum (dogs and rabbits); while those from the gall-bladder and the center of gallstones commonly produce cholecystitis. The bacillus isolated from erythema nodosum when injected as isolated showed a marked affinity for the subcutaneous tissue in dogs, rabbits and guinea-pigs, producing localized hemorrhages followed by infiltration resembling the nodes seen in man, the lesions often being symmetrically placed usually near a relatively large blood-vessel. On artificial cultivation and on animal passage the strains lost this peculiar property. In both instances the organism tended to become a streptococcus and on passage it acquired an affinity for the joints, fascia, muscles and endocardium. This fact would seem to afford an explanation of the common occurrence of arthritis and sometimes endocarditis in erythema nodusum and allied conditions.

The almost constant presence of diphtheroid organisms in the glands and their demonstration in the blood in Hodgkin's disease in conjunction with the results of experiments on monkeys by Bunting and Yates and by Negri and Mieremet, and the good results obtained in some cases by the use of autogenous vaccines suggest strongly that these organisms play at least a prominent rôle in this disease; but the fact that other organisms are commonly found also and that diphtheroid bacilli indistinguishable from some of the strains from Hodgkin's disease are found in many other conditions compels one to wait for more experimental evidence before concluding that these bacilli are the real cause of Hodgkin's disease.

The etiologic rôle of infection in goiter is becoming more apparent; enlargement of the thyroid gland has been noted in various infections, particularly in rheumatism; and the peculiar distribution of endemic goiter, and other clinical facts, suggest that the cause of simple and exophthalmic goiter may be due to an infection. The symptoms and the enlargement of the gland, however, have usually been considered toxic in nature and not due to localization of the bacteria in the gland. No one, as far as I can learn, has isolated an organism from the thyroid in a series of cases. The fact that the same organism has been isolated from goiters in both dogs and man, and in the latter apparently in proportion to the degree of symptoms and from the blood, the absence of this organism in normal thyroid glands in dogs, the fact that it lives longer in the thyroid gland than in the kidney and other tissues of dogs following intravenous injection, and that goiter has appeared in two dogs after intravenous injection of this organism, suggest that goiter may be due not only to toxins produced in other regions of the body but to an actual localization in the gland of bacteria. Studies of the chief organism found show that it is probably closely related to the streptococcus group and this may explain why thyroid enlargement is observed commonly in rheumatism and in cases having foci of infection in which the predominating flora is streptococcal.

The significance of the findings of the streptococcus in cystic ovaries is not yet clear. Further experiments on animals are necessary and the immunologic reactions in the various conditions, particularly in goiter and cystic ovaries, need to be worked out before definite conclusions can be drawn. It may be that the organisms isolated are merely secondary invaders. The rather frequent occurrence of diphtheroid-like organisms in the tissues and blood during life is, I believe, significant. They are probably not wholly secondary or harmless invaders.

Altogether the $B$. welchii, or an organism closely related, has been isolated from various tissues in man 103 times and from the blood or joint fluids six times. It has frequently been found in various tissues, such as the thyroid, muscle and kidney of dogs and occasionally in livers of rabbits and guinea-pigs. On account of this surprising finding and because the organism is an anaerobe and spore-bearing, every possible source, especially the serum and ascitic fluids, other than the tissue inoculated has been excluded by control-tests. The common presence of this organism in the tissues of man and the dog (carnivorous animals) and its absence in the rabbit and guinea-pig (herbivorous animals) is, I believe, of significance. It exists in the tissues probably chiefly in the spore form and the growth usually does not result except when the oxygen pressure has reached a certain grade. The fact that the strains grow readily at a certain grade of oxygen pressure without production of gas and that 
they produce the characteristic growth with gas formation only under strict anaerobic conditions suggest that under special circumstances (a lowered oxygen pressure, increased virulence or growth in symbiosis with other bacteria perhaps) $B$. weelchii may not be an entirely harmless invader.

Finally, whatever the ultimate significance of these findings may prove to be, the common isolation of bacteria from tissues which have been considered sterile is of definite importance, lends support to the contentions of Adami and others that certain ill-understood conditions are due to a low-grade infection and not merely intoxication and calls for an extensive study of the whole question by this or similar methods.

I wish to express my very great appreciation to the numerous physicians, particularly Dr. Billings, who have supplied me with material. The more complete reports are to appear in the Journal of Infectious Diseases.

\section{South Wood Street.}

\section{ABSTRACT OF DISCLSSION}

ON PAPERS OF DRS. BILLINGS AND ROSENOW

Dr. William S. Thayer, Baltimore: There has been no one circumstance in the last fifteen years that has so changed the aspect of the practice of medicine as the recognition of the influence of local foci of infection on the production and course of a variety of general processes of previously uncertain etiology. In the discussion on cardiac disease, Dr. Cabot, in his classification, referred to the "rheumatic" cases stating that although the term was rough and general, it yet covered those infections which are commonly associated with arthritis as well as cardiac disease. It is well to emphasize this association. When one meets to-day a mitral stenosis or insufficiency he proceeds in a very different way from that in which he did twenty years ago. One's first thought now should be to search for a focus of chronic infection by the removal of which the probability of progression of the malady may be greatly reduced. The proportion of cases of valvular disease of uncertain origin that show old chronic affections of sinuses, of teeth, especially of the tonsils, is very large. I would emphasize also that in tonsilar operations it is most important that the operation be complete. In a very large number of patients who come to me with the statement that their tonsils have been removed, it is easy to demonstrate that most of the lower pole of the tonsil is still present, the crypts often sealed by cicatricial tissue and more dangerous perhaps than before operation. Even in the hands of men whose technic is very good it is no uncommon to see in young people a hyperplasia of small islands of lymphoid tissue which remained after operation. These little islands may later be infected and give rise to all the symptoms formerly experienced.

Dr. A. C. GRiffith, Kansas City, Mo.: It has been my good fortune since the discovery of the diphtheroid bacilli to see a few cases of Hodgkin's disease. One case in particular occurred in an elderly lady who had been suffering for six or seven years with glandular enlargement. We removed one of these glands and developed the diphtheroid bacillus; also a coccus. Dr. Rosenow has described their clinical characteristics. These organisms are mostly of the aerobic type. The patient was put on autogenous vaccines made from the growth of the organism. The treatment was given in gradually increasing doses until the swelling of the glands began to disappear and the patient was doing nicely. She, however, was persuaded to take up Christian Science. Two weeks later I was called in when she was dying from overinfection or intoxication as a result of the activity of the glands. Necropsy showed that the changes in the glands were those characteristic of Hodgkin's disease; there was the degeneration and softening in the center and the outer harder portion. We used the vaccines three hundred millions to the cubic centimeter. I started in with 0.5 c.c. and increased the dose to 2 c.c. I gave first a dose every three or four days and toward the last 2 c.c. every other day, and the glands were disappearing when the patient left me to follow one of the cults.

Dr. Frank Smithies, Chicago: In the study of the histories of nearly ten thousand cases of gastro-intestinal trouble I have been struck by the fact that aggravations of the disturbances were quite common in the spring and the fall. It will be especially noted that this happens in the case of peptic ulcer. It will also be recalled that these seasons of the year include the periods during which epidemic infectious diseases are most prevalent. In view of Dr. Rosenow's investigations, infection of the gastric mucosa by microorganisms might occur at these periods during the course of the disease. In the case of ulcers already existing, reinfection may occur and the process be lighted up. The acid reaction of the stomach is no criterion of its bacterial cleanliness. I have shown this by my study of the findings in more than 7,000 consecutive test-meals. In the majority of the gastric-ulcer cases, there is a possibility of finding foci of infection elsewhere. Studying 1,500 peptic ulcers, I find that in more than 60 per cent. coincident or earlier infection of the gall-bladder or appendix could be noted. In cases of cholecystitis, with or without stones, there was pancreatitis in 12.5 per cent., and in common-duct obstruction, pancreatitis was noted in more than 28 per cent. of instances. It should be emphasized that cleaning up a local focus, as in the mouth, does not guarantee that patients will recover from damage that has already been done in the extensive area of the gastro-intestinal tract.

Dr. E. Libman, New York: The occurrence of exacerbations in cases of duodenal ulcer following so-called colds has been particularly emphasized by Hershell of London. In trying to establish a diagnosis between duodenal ulcer and cases of functional disturbance a history of such occurrence is of value. I was interested in Dr. Rosenow's statement that he is not willing definitely to accept the organism described in connection with Hodgkin's disease as being the cause of the disease. Dr. Celler has made a study of a number of cases of various forms of diseases of lymph-nodes and has found the bacillus not only in cases of Hodgkin's disease, but also in other diseases of the lymph-nodes, and particularly in two cases of typical tuberculosis. Apart from that, the Streptococcus viridans has been found in lymphnodes in cases of Hodgkin's disease as well as in other lymph-nodes. Complement-fixation tests made by Dr. Olitsky in cases of Hodgkin's disease have proved to be absolutely negative in all instances. These findings lead us to be careful before accepting the organism as being the etiologic factor in the disease and make us keep in mind the question of latent microbism. There is no doubt that Dr. Rosenow has produced definite mutations in the streptococcus-pneumococcus group. As regards Dr. Rosenow's statement that the organisms from a certain type of infection cause, at least for a time, the same lesions in animals as in man, some work done by Drs. Rothschild and Thalhimer indicate that exceptions occur. They succeeded in 50 per cent. of the inoculated animals, in producing arthritis with recently inoculated strains obtained from cases of subacute endocarditis in man. Pericarditis has been found in animals inoculated with the same organisms and this is a very unusual phenomenon in the disease from which the organisms are cbtained.

Dr. James M. Anders, Philadelphia: These two papers rivet the attention of the profession especially on the head as the seat of infective foci. It has long been well understood that in the case of acute miliary tuberculosis invariably an old focus exists somewhere in the body, most frequently in the lungs, from which the tubercle bacilli come. These papers emphasize the importance of a careful inspection of the mouth and throat as a part of our routine examinations of patients. If foci of inflammation are found there, they should, of course, be energetically attacked without delay. It is important to recollect that if removal of such focal infections fail to bring about a cure of the condition from. which the 
patient may be suffering, it is not evidence to the contrary, but means, as pointed out by Dr. Billings, that hematogenous metastasis has already taken place. The American Medical Association has its Section on Stomatology, and it would be entirely proper, it seems to me, for the Section on Practice of Medicine to urge, through the House of Delegates the establishment of chairs of Stomatology in our medical colleges.

Dr. De Lancey Rochester, Buffalo, N. Y.: I wish to speak of focal infections especially of the teeth and tonsils. In three cases having teeth infections accompanied by endocarditis in which there were no manifest symptoms in the mouth, but in which the Roentgen ray showed an abscess at the roots of the teeth, when the abscess was removed the symptoms subsided and the patients improved clinically. The organism found was the ordinary streptococcus pyogenes. I have had four cases which have impressed on me the importance of having the tonsils completely removed in cases of infection. The danger of an incomplete operation is illustrated in one case in which the toxic process had gone too far before the trouble was recognized. The patient was a woman who gave a history of rheumatism, multiple arthritis and endocarditis which had been treated in the hospital for two weeks. She improved under the salicylates and got up. Later she had a recurrence, ushered in with a temperature of $104.5 \mathrm{~F}$. and her pulse was uncountable. She gave a history of having had a tonsil operation ten years before. There was also a history of sore throat and an exudate over the tonsils. The condition of the patient was so serious that she could not be operated on. She died of severe systemic toxemia, in all probability due to infection from the tonsils.

Dr. Alfred Stzngel. Philadelphia: Dr. Rosenow's statement with regard to the cause or the part played by the organism in Hodgkin's disease was very conservative. $\mathrm{He}$ said that we are not sure of the relation of the diphtheroid organism to Hodgkin's disease, but it seems to me that we are sure that this organism is the specific organism of Hodgkin's disease. I have only four cases of such infection to report: one of endothelioma; chronic simple inflammation of the glands; one of lymphoid enlargement in a patient with rheumatoid arthritis, and lastly another rheumatoid case not easily classified.

Dr. J. Birney Guthrie, New Orleans: Has Dr. Rosenow applied his technic in finding the cause of the short fevers of obscure origin in children, which are accompanied by lymphatic enlargement-tho so-called glandular fevers? These symptoms of unexplained fever and lymphatic enlargement are quite common in children and it would be interesting to have this method applied to their investigation.

Dr. Harvey D. Wood, Fayetteville, Ark.: Owing to the rapid progress that has been made in our knowledge of the cause of inflammation and infection, I believe the time has come when the definition of the termination "itis" should be made to include infection as well as inflammation. Since listening to Dr. Billings' paper and the following discussion I have been more than ever fully impressed with the need of a more comprehensive definition of this termination. The phenomena of seasonal recurrences and exacerbations of ulcer, due to infection, have been so clearly set forth in this discussion that it gives us a better understanding of many pathologic processes. The infection that comes from the bite of the infected bedbug is now thought by some, who are doing special work on this line, to be the cause of pellagra. This disease has its period of exacerbation in the springtime, when this pestiferous insect begins to get busy. Should this prediction prove to be the true source of the pathology of this disease, there are many persons whose minds will be relieved from the fear of having this malady.

Dr. Frank Billings, Chicago: In our group of workers we know that Dr. Rosenow is dependable. He has an opportunity to study disease clinically. The diphtheroid bacillus has been successfully cultivated from the enlarged lymphnodes of about forty, and from the blood of five patients with Hodgkin's disease. These five had fever. The histology of the glandular tissues varied from typical Hodgkin's to simple lymphocytomas, lymphosarcoma and tuberculosis. In one lymphosarcoma the organism could not be cultivated. Many of these patients have received the autogenous vaccine subcutaneously in the dosage of $10,000,000$ to $1,500,000,000$. usually repeated once a week. Two have received the vaccine in large doses intravenously. Used subcutaneously there is no recognizable general reaction. Intravenously, general reaction has been noted; fever, rapid pulse, general discomfort, headache, etc. Practically all patients have also been treated with efficient roentgenization. In the chronic nonfebrile cases the vaccines and roentgenotherapy seem to procluce better results than the Roentgen ray alone. We are now preparing a report on the series of patients. Dr. L. Hektoen will report the histopathology of the glands, Dr. Rosenow will give the bacteriology and I shall prepare the clinical data. We are now attempting to immunize a horse with mixed strains of the diphtheroid bacillus. If successful we shall add the serum to the treatment.

Dr. E. C. Rosenow, Chicago: One word as to the importance of foci of infection other than those of the mouth. I recently saw a patient in Dr. Billings' service who had a rheumatic endocarditis, pericarditis, etc., which apparently was the result of an infected ingrowing toe-nail. The toe was operated on, the streptococcus isolated and the disease reproduced in dogs and rabbits. Another patient had a severe rheumatism including pericarditis and endocarditis which began ten days after a crushing injury of the thumb. Again the disease was reproduced with the streptococcus isolated from the wound on the thumb. I am sorry that I am not prepared to say what the cultures of the glands show in the short fevers in children with glandular enlargement. I believe that the glands in these cases contain bacteria which are the cause of the disease. Streptococci were isolated from the glands in seven of the cases of Hodgkin's disease. It is quite well known that ulcers heal more quickly in the cardiac end of the stomach than in the pyloric end or duodenum. This is generally believed to be due to mechanical causes, but if streptococci play a róle in the etiology of ulcer, as seems likely, why might not this be due in part to a greater bactericidal power of the mucous membrane of the former because acid-secreting, than that of the pylorus and duodenum in which no acid cells are present? The infectious nature of ulcer would best explain the seasonal exacerbations and the recurrences which come without known cause, the recurrences taking place when the immunity of the blood from a previous attack has disappeared. The most important factor in the management of ulcer of the stomach-the recognition and removal of various foci of infection-has been overlooked in the past. Proper regard of this point should reduce the incidence, percentage of recurrences and earlier healing of ulcer of the stomach and duodenum.

Good Roads Prevent Disease.-Few persons, on first thought, would see any possible connection between good roads and good health. Yet the State Board of Health of Kansas says that good roads can and will prevent disease. How? By the removal of weeds and trash. Weeds and trash prevent the prompt evaporation of moisture and promote retention of ground water. These make ideal breeding spots for mosquitoes, flies and other disease carriers.

Good roads also prevent disease by providing good drainage. Many farms have no means of drainage except by ditches along roadways. Open ditches, clear of brush and debris, with hardened surface and proper fall, afford these farms the opportunity of ridding themselves of many a stagnant pool. . . . Road-oiling in itself is destructive of insect larvae, especially mosquitoes-a well-known fact. Dry roads offer pedestrians and notably children who are compelled to walk to and from school, dry shoes and feet. . . Good roads are active disease prevention agencies, aside from their financial and commercial value.-Monthly Bull. Ohio State Board of Health. 